\title{
Hydrazide-Hydrazones in the Synthesis of 1,3,4-Oxadiazine, 1,2,4-Triazine and Pyrazole Derivatives with Antitumor Activities
}

\author{
Rafat M. Mohareb*,a,b Rehab A. Ibrahim ${ }^{\mathrm{c}}$ and Hosam E. Moustafa ${ }^{\mathrm{d}}$ \\ ${ }^{a}$ Faculty of Pharmacy, Organic Chemistry Department, October University of Modern Sciences \& Arts, October City, A. \\ R. Egypt \\ ${ }^{b}$ Faculty of Science, Chemistry Department, Cairo University, Giza, A.R. Egypt \\ ${ }^{c}$ Chemistry Department, Faculty of Engineering, New Cairo Academy, El-Tagemmoe El-Khames, Egypt \\ ${ }^{d}$ Applied Medical Sciences Department, Community College in Quaryat, Al jouf University, Saudi Arabia
}

\begin{abstract}
The reaction of cyanoacetyl hydrazine (1) with the $\alpha$-haloketones $\mathbf{2 a - 2 c}$ in 1,4-dioxane afforded the hydrazidehydrazone derivatives 3a-3c. The latter products were used in a series of heterocyclization to give 1,3,4-oxadiazine, 1,2,4triazine and pyrazole derivatives. All compounds synthesized through this work are new products and their antitumor evaluation towards three human tumor cell lines representing different tumor types, namely, breast adenocarcinoma (MCF-7), non-small cell lung cancer (NCI-H460) and CNS cancer (SF-268). Some of the synthesized products showed high inhibitory effect towards the three cell lines.
\end{abstract}

Keywords: Antitumor, hydrazide-hydrazone, 1,3,4-oxadiazine, 1,2,4-triazine, pyrazole.

\section{INTRODUCTION}

It is well known that the hydrazone group plays an important role for the antimicrobial activity. Thus, a number of hydrazide-hydrazone possessed interesting antibacterialantifungal [1-4], anticonvulsant [5-7], anti-inflammatory [8,9], antimalarial [10] and antituberculosis activities [1117]. With the aim of obtaining new hydrazide-hydrazones with such wide spectrum of pharmaceutical applications, we report here the synthesis of a series of hydrazide-hydrazones 3a-3c via the reaction of cyanoacetylhydrazine 1 with $\alpha$ bromoketones (2a-2c). Moreover, the synthesized hydrazidehydrazone derivatives were used in a series of heterocyclic transformations to give 1,3,4-oxadiazine, 1,2,4-triazine and pyrazole derivatives [18-21].

\section{RESULTS AND DISCUSSION}

The reaction of cyanoacetyl hydrazine 1 with the $\alpha$ haloketones $\mathbf{2 a - 2 \mathbf { c }}$ in 1,4-dioxane at room temperature gave the $\alpha$-bromohydrazone derivatives 3a-3c (Scheme 1). The structures of the latter were elucidated through analytical and spectral data. Thus, the ${ }^{1} \mathrm{H}$ NMR spectrum of 3a showed the presence of two singlets at $4.77 .5 .38 \mathrm{ppm}$ corresponding to two $\mathrm{CH}_{2}$ groups, a multiplet at 7.27-7.40 ppm corresponding to phenyl protons and a singlet at $8.88 \mathrm{ppm}\left(\mathrm{D}_{2} \mathrm{O}\right.$ exchangeable) for an $\mathrm{NH}$ group. Moreover, the ${ }^{13} \mathrm{C}$ NMR of 3a showed the presence of $\delta: 27.0,64.6$ corresponding to the two $\mathrm{CH}_{2}$ groups together with other signals which are consistent with the proposed structure (see experimental section).

*Address correspondence to this author at the Faculty of Pharmacy, Organic Chemistry Department, October University of Modern Sciences \& Arts, October City, A. R. Egypt; Tel: 002012-3406896; Fax: 002025676570;

E-mail: raafat_mohareb@yahoo.com
Compounds 3a-3c underwent ready cyclization when heated in a boiling water bath with sodium ethoxide in ethanol solution to form the 1,3,4-oxadiazine derivatives $\mathbf{4 a - 4} \mathbf{c}$ (Scheme 2). The analytical and spectral data obtained for the synthesized compounds were are in agreement with the proposed structures (see experimental section).

The reaction of 3a with either hydrazine hydrate $\mathbf{5 a}$ or phenylhydrazine $\mathbf{5 b}$ gave the 1,2,4-triazine derivatives $\mathbf{7 a}$ or $\mathbf{7 b}$, respectively (Scheme 3). Formation of the latter is explained in terms of the non-isolable intermediate formation of $6 \mathbf{a}$ or $\mathbf{6 b}$ followed by dehydration. The elemental analysis and ${ }^{1} \mathrm{H}$ NMR spectra were the basis of structure elucidation.

The 1,2,4-triazine derivatives $\mathbf{7 a}$ and $\mathbf{7 b}$ bearing the cyanomethyl group showed a high reactivity towards aromatic aldehydes. Thus, the reaction of compounds $\mathbf{7 a}$ and $\mathbf{7 b}$ with benzaldehyde 8 gave the derivatives 9a and 9b (Scheme 4). On the other hand, their reaction with salicylaldehyde $\mathbf{1 0}$ gave the coumarin derivatives 11a and 11b.

The reaction of compounds 3a-3c with benzaldehyde gave the 2-benzalcyanomethyl-1,3,4-oxadiazine derivatives 13a-13c (Scheme 5). The structures of the obtained products were elucidated on the basis of analytical and spectral data. The same compounds 13a-13c, respectively were obtained through reaction of $\mathbf{4 a - 4} \mathbf{c}$ with benzaldehyde.

Compounds 3a-3c bearing the $\alpha$-bromomethyl group seemed to be reactive towards nucleophilic reagents. Thus, their reaction with potassium cyanide gave the pyrazole derivatives 15a-15c (Scheme 6). Formation of these products was based on the formation of the non isolable intermediates 14a-14c followed by cyclization. Our trials to isolate the acyclic intermediates failed. The analytical and spectral data of compounds 15a-15c were in agreement with the proposed structures. Thus, the ${ }^{1} \mathrm{H}$ NMR spectrum of 15a showed a 

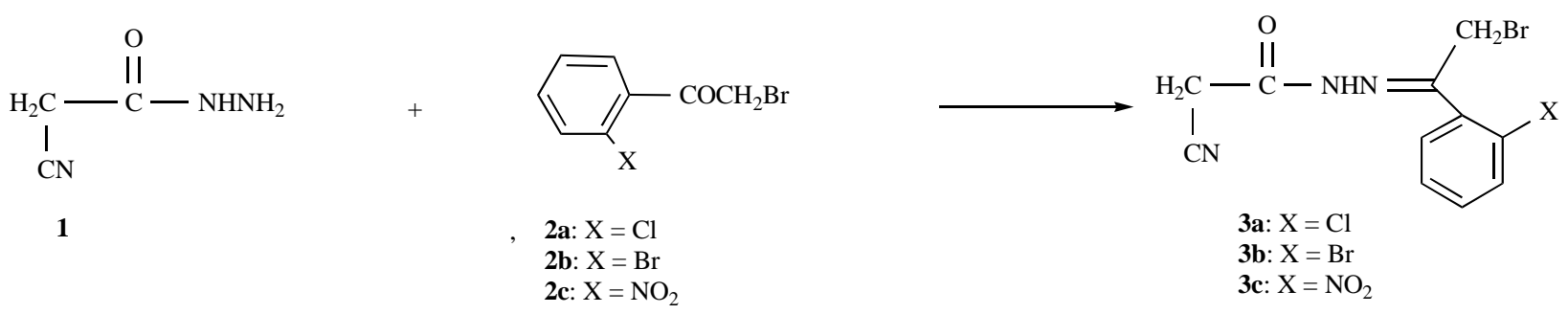

Scheme 1. Reagents and conditions: 1,4-dioxane (0.01 mole equiv.), heat 2h, stirring at r.t. 2 h, yield $80 \%$ (3a), $72 \%$ (3b), $88 \%$ (3c).

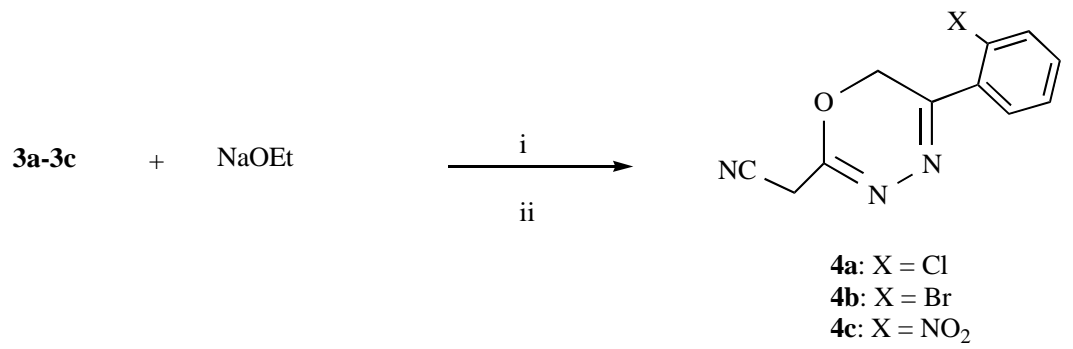

Scheme 2. Reagents and conditions:( i) NaOEt, EtOH (0.01 equiv.), heat in a boiling water bath $4 \mathrm{~h}$ (ii) $\mathrm{HCl}$ in ice/water, $\mathrm{pH} 7,70 \%$ (4a), 78 $\%(4 \mathbf{b}), 63 \%(\mathbf{4 c})$.

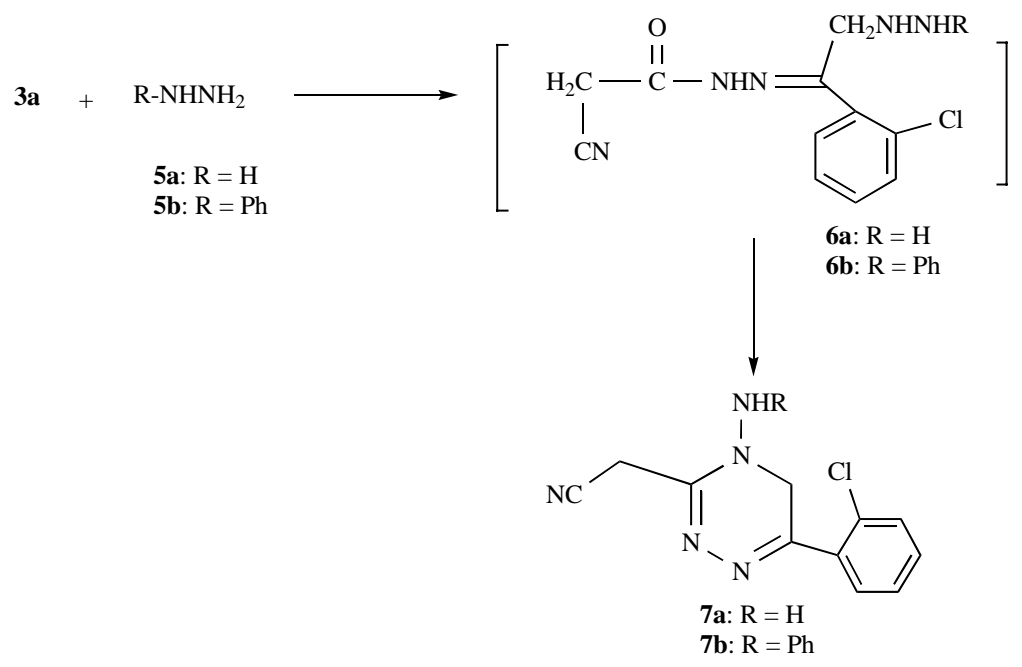

Scheme 3. Reagents and conditions: 1,4-dioxane (0.01 equiv.), heat 3 h, ice/water with $\mathrm{HCl}, 60 \%$ (7a), $68 \%$ (7b).
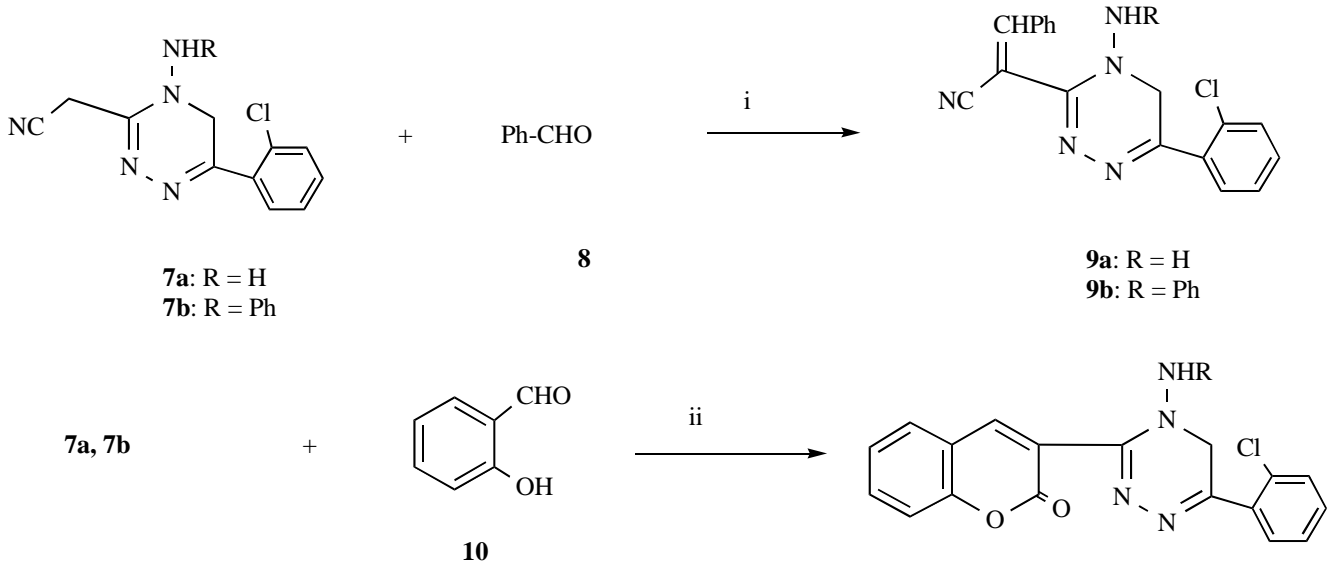

11a: $\mathrm{R}=\mathrm{H}$

11b: $\mathrm{R}=\mathrm{Ph}$

Scheme 4. Reagents and conditions: (i) $\mathrm{NH}_{4} \mathrm{OAc}$, Oil bath $120^{\circ} \mathrm{C}, 15$ min. (0.01 equiv.), EtOH, $72 \%$ (9a), $69 \%$ (9b). (ii) 1,4-dioxane (0.01 equiv.), piperidine, heat $4 \mathrm{~h}, \mathrm{EtOH}, 62 \%$ (11a), $57 \%$ (11b). 
singlet at 4.64 ppm corresponding to $\mathrm{CH}_{2}$ group, a singlet at 4.90 ppm corresponding to an $\mathrm{NH}_{2}$ group, a singlet at 6.52 ppm corresponding to the pyrazolic proton $\mathrm{H}-4$ and a multiplet at 7.28-7.34 ppm for the protons of $\mathrm{C}_{6} \mathrm{H}_{4}$ group. Moreover, the ${ }^{13} \mathrm{C}$ NMR showed signals that are in agreement with the structure of $\mathbf{1 5 a}$ (see experimental section).

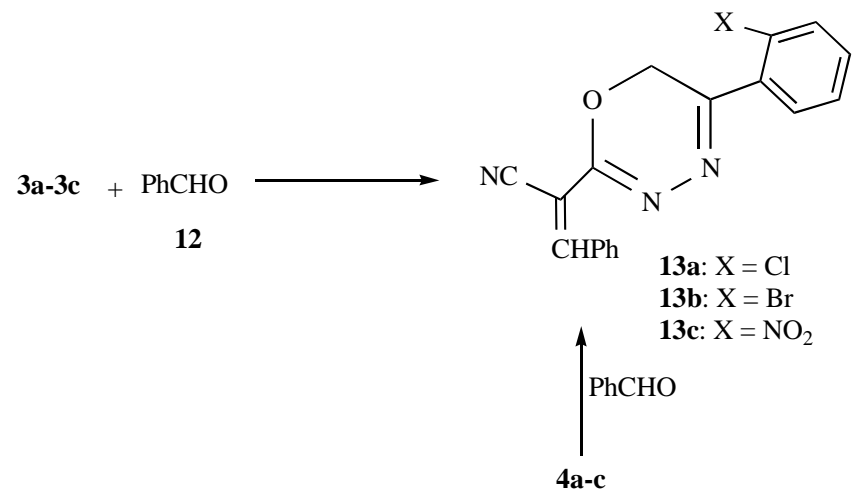

Scheme 5. Reagents and conditions: 1,4-dioxane (0.01 mole equiv.), piperidine, heat $6 \mathrm{~h}, \mathrm{HCl}$ in ice/water, $63 \%$ (13a), $66 \%$ (13b), $70 \%$ (13c).

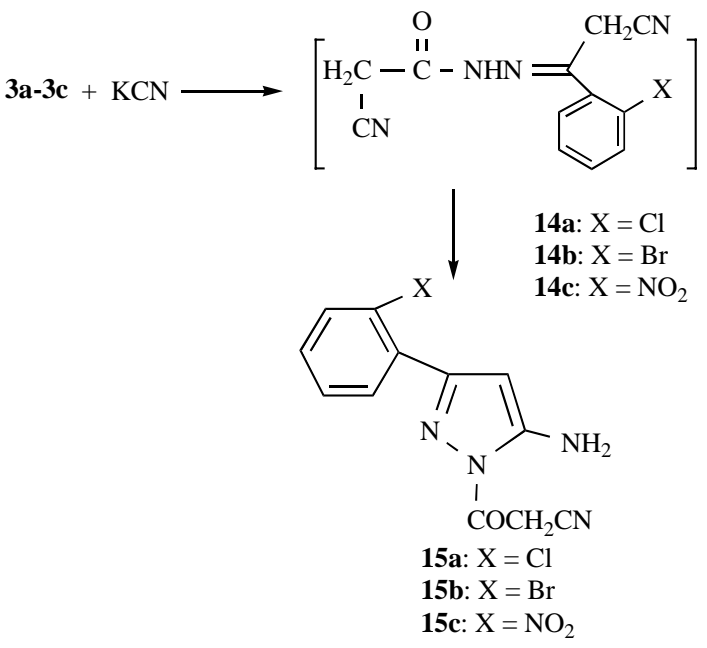

Scheme 6. Reagents and conditions: EtOH (0.01 mole equiv.), water bath at $60{ }^{\circ} \mathrm{C}, 0.5 \mathrm{~h}, \mathrm{HCl}$ in ice/water $\mathrm{pH} 6,74 \%$ (15a), $70 \%$ (15b), $64 \%$ (15c).

\section{Effect of the Synthesized Compounds on the Growth of Human Tumor Cell Lines}

The effect of compounds 3a-15c was evaluated on the in vitro growth of three human tumor cell lines representing different tumor types, namely, breast adenocarcinoma (MCF-7), non-small cell lung cancer (NCI-H460) and CNS cancer (SF-268), after a continuous exposure of $48 \mathrm{~h}$. The results are summarized in Table $\mathbf{1}$.

All the tested compounds were able to inhibit the growth of the human tumor cell lines in a dose-dependent manner (data not shown). The 1,3,4-oxadiazine derivative $\mathbf{4 c}$, the 1,2,4-triazine derivative 11a and the pyrazole derivative 15c showed the highest inhibitory effect of the tested compounds, exhibiting an equivalent potency in all the three tumor cell lines. However, their activity is largely lesser than the positive control. While compounds $\mathbf{3 b}, \mathbf{3 c}, \mathbf{7 a}, \mathbf{9 a}, \mathbf{1 3 b}$, 15a showed moderated growth inhibitory effect relative to compounds 4c, 11a and 15c. On the other hand, compounds 3a, 4a, 4b, 9b, 11b, 13a and 15b showed the lowest inhibitory effect relative to the tested compounds. It is convenient to observe that the pyrazole derivative $\mathbf{1 5 c}$ with its $o$ nitrophenyl group showed the maximum inhibitory effect through the three cell lines. Comparing the activities of compounds $\mathbf{4 a}, \mathbf{4 b}$ and $\mathbf{4 c}$, it is observed that the $o$-chlorophenyl1,3,4-oxazine 4a presents a weaker growth inhibitory effect while the o-nitrophenyl-1,3,4-oxazine 4c showed high inhibitory effect although the results in NCI-H460 cell line are comparable.

\section{EXPERIMENTAL SECTION}

\section{General Information}

Melting points were determined in open capillaries and are uncorrected. I.R spectra were measured using $\mathrm{KBr}$ discs on a Pye Unicam SP-1000 spectrophotometer. ${ }^{1}$ HNMR \& ${ }^{13} \mathrm{C}$ NMR spectra were measured on a Varian EM390-200 $\mathrm{MHz}$ instrument in DMSO- $\mathrm{d}_{6}$ as solvent and using TMS as internal standard, and chemical shifts $(\delta)$ are expressed in ppm. Elemental analyses were determined on a Yanaco CHN Corder elemental analyzer (Japan).

$\alpha$-Bromo(o-chloroacetophenone)- $\alpha$-cyanoacetylhydrazone $3 a, \quad \beta$-bromo(o-bromoacetophenone)- $\alpha$-cyanoacetylhydrazone $3 b$ and $\beta$-bromo(o-nitroacetophenone)- $\alpha$-cyanoacetylhydrazone $3 c$

To a solution of cyanoacetyl hydrazine 1 (0.99 g, 0.01 $\mathrm{mol})$, in 1,4-dioxane $(40 \mathrm{~mL})$ either of $o$-chlorophenacylbromide $2 \mathbf{a}(2.3 \mathrm{~g}, 0.01 \mathrm{~mol}), o$-bromophenacylbromide $\mathbf{2 b}$ $(2.80 \mathrm{~g}, 0.01 \mathrm{~mol})$ or o-nitrophenacylbromide $(2.46 \mathrm{~g}, 0.0 .1$ mol) was added. The reaction mixture was kept at room temperature with stirring for $2 \mathrm{~h}$ and the formed solid product was filtrated off.

Compound 3a: Pale yellow crystals were recovered from ethanol in a yield of $80 \%(2.49 \mathrm{~g})$; mp: $177-179^{\circ} \mathrm{C}$. I.R $\left(\mathrm{v} / \mathrm{cm}^{-1}\right)=3480-3334(\mathrm{NH}), 3054(\mathrm{CH}$ aromatic $), 2887$ $\left(\mathrm{CH}_{2}\right), 2260(\mathrm{CN}), 1684(\mathrm{C}=\mathrm{O}), 1666(\mathrm{C}=\mathrm{N}), 1641(\mathrm{C}=\mathrm{C})$. ${ }^{1} \mathrm{H}$ NMR (200 MHz, DMSO-d 6 ) $\delta 4.77\left(\mathrm{~s}, 2 \mathrm{H}, \mathrm{CH}_{2}\right), 5.38(\mathrm{~s}$, $\left.2 \mathrm{H}, \mathrm{CH}_{2}\right)$ 7.27-7.40 (m, 4H, $\left.\mathrm{C}_{6} \mathrm{H}_{4}\right), 8.88(\mathrm{~s}, 1 \mathrm{H}, \mathrm{NH}) .{ }^{13} \mathrm{C}$ NMR (200 MHz, DMSO-d $) \delta 27.0\left(\mathrm{CH}_{2}\right), 64.6\left(\mathrm{CH}_{2}\right), 116.3$ $(\mathrm{CN}), \quad 126.8, \quad 127.0, \quad 128.7,130.0,138.4\left(\mathrm{C}_{6} \mathrm{H}_{5}\right), 157.3$ $(\mathrm{C}=\mathrm{N}), 172.7(\mathrm{C}=\mathrm{O})$. Calculated for $\mathrm{C}_{11} \mathrm{H}_{9} \mathrm{BrClN}_{3} \mathrm{O}: 314.57$, $\mathrm{C}: 42.00, \mathrm{H}: 2.88, \mathrm{~N}: 13.36)$. Found, $\mathrm{C}: 42.09, \mathrm{H}: 3.11, \mathrm{~N}$ : 13.40 .

Compound 3b: Pale yellow crystals were recovered from ethanol in a yield of $72 \%(2.59 \mathrm{~g})$; mp: $202-205^{\circ} \mathrm{C}$. I.R $\left(\mathrm{v} / \mathrm{cm}^{-1}\right)=3463-3329(\mathrm{NH}), 3050(\mathrm{CH}$ aromatic $), 2887$ $\left(\mathrm{CH}_{2}\right), 2260(\mathrm{CN}), 1690(\mathrm{C}=\mathrm{O}), 1666(\mathrm{C}=\mathrm{N}), 1636(\mathrm{C}=\mathrm{C})$. ${ }^{1} \mathrm{H}$ NMR (200 MHz, DMSO-d $\left.{ }_{6}\right) \delta 4.74\left(\mathrm{~s}, 2 \mathrm{H}, \mathrm{CH}_{2}\right), 5.40(\mathrm{~s}$, $\left.2 \mathrm{H}, \mathrm{CH}_{2}\right), 7.26-7.35\left(\mathrm{~m}, 4 \mathrm{H}, \mathrm{C}_{6} \mathrm{H}_{4}\right), 8.83(\mathrm{~s}, 1 \mathrm{H}, \mathrm{NH}) .{ }^{13} \mathrm{C}$ NMR (200 MHz, DMSO-d $\left.)_{6}\right) \delta 27.2\left(\mathrm{CH}_{2}\right), 64.5\left(\mathrm{CH}_{2}\right), 116.0$ $(\mathrm{CN}), 126.0,126.8,127.0,128.7,133.0,139.4\left(\mathrm{C}_{6} \mathrm{H}_{5}\right), 157.0$ $(\mathrm{C}=\mathrm{N}), 172.9(\mathrm{C}=\mathrm{O})$. Calculated for $\mathrm{C}_{11} \mathrm{H}_{9} \mathrm{Br}_{2} \mathrm{~N}_{3} \mathrm{O}: 359.02$, C: $36.80, \mathrm{H}: 2.53, \mathrm{~N}: 11.70$. Found, C: $36.66, \mathrm{H}: 2.49, \mathrm{~N}$ : 11.38 .

Compound 3c: Orange crystals were recovered from ethanol in a yield of $88 \%(2.84 \mathrm{~g})$; mp: $210-214^{\circ} \mathrm{C}$. I.R $\left(\mathrm{v} / \mathrm{cm}^{-1}\right)=3465-3326(\mathrm{NH}), 3056(\mathrm{CH}$ aromatic $), 2888$ 
Table 1. Effect of Compounds 3-15c on the Growth of Three Human Tumor Cell Lines

\begin{tabular}{|c|c|c|c|}
\hline Compound No. & \multicolumn{3}{|c|}{$\mathbf{G I}_{50}(\mu \mathbf{M})$} \\
\hline $3 a$ & $66.6 \pm 12.2$ & $12 \pm 6.2$ & $24.8 \pm 3.2$ \\
\hline $3 \mathbf{b}$ & $20 \pm 0.4$ & $24.3 \pm 0.8$ & $32 \pm 0.8$ \\
\hline $3 c$ & $30 \pm 0.6$ & $17.3 \pm 1.4$ & $22.3 \pm 1.5$ \\
\hline $4 b$ & $72.7 \pm 17.5$ & $40.2 \pm 12.8$ & $50.0 \pm 9.01$ \\
\hline $4 c$ & $11.8 \pm 0.6$ & $14.5 \pm 0.8$ & $16.7 \pm 1.6$ \\
\hline $7 a$ & $35.4 \pm 10.2$ & $24.1 \pm 0.8$ & $18.9 \pm 6.8$ \\
\hline $7 b$ & $38.0 \pm 1.8$ & $44.0 \pm 0.8$ & $20.5 \pm 1.1$ \\
\hline $11 \mathrm{a}$ & $11.9 \pm 0.5$ & $14.1 \pm 0.6$ & $20.3 \pm 0.5$ \\
\hline $11 \mathrm{~b}$ & $70.9 \pm 0.9$ & $43.6 \pm 1.8$ & $56.8 \pm 0.8$ \\
\hline 13a & $66.6 \pm 16.9$ & $38.9 \pm 10.8$ & $50.8 \pm 8.6$ \\
\hline $13 b$ & $40.6 \pm 12.2$ & $32.6 \pm 8.6$ & $60.4 \pm 14.8$ \\
\hline $13 c$ & $22.0 \pm 0.2$ & $30.6 \pm 1.4$ & $38.4 \pm 0.6$ \\
\hline $15 a$ & $20 \pm 0.4$ & $24.3 \pm 0.8$ & $32 \pm 0.8$ \\
\hline $15 \mathrm{~b}$ & $70.9 \pm 0.9$ & $43.6 \pm 1.8$ & $56.8 \pm 0.8$ \\
\hline
\end{tabular}

Results are given in concentrations that were able to cause $50 \%$ of cell growth inhibition $\left(\mathrm{GI}_{50}\right)$ after a continuous exposure of $48 \mathrm{~h}$ and show means \pm SEM of three-independent experiments performed in duplicate.

$\left(\mathrm{CH}_{2}\right), 2263(\mathrm{CN}), 1685(\mathrm{C}=\mathrm{O}), 1661(\mathrm{C}=\mathrm{N}), 1634(\mathrm{C}=\mathrm{C})$. ${ }^{1} \mathrm{H}$ NMR $\left(200 \mathrm{MHz}, \mathrm{DMSO}-\mathrm{d}_{6}\right) \delta 4.79\left(\mathrm{~s}, 3 \mathrm{H}, \mathrm{CH}_{2}\right), 5.44(\mathrm{~s}$, $\left.2 \mathrm{H}, \mathrm{CH}_{2}\right), 7.31-7.39\left(\mathrm{~m}, 4 \mathrm{H}, \mathrm{C}_{6} \mathrm{H}_{4}\right), 8.80(\mathrm{~s}, 1 \mathrm{H}, \mathrm{NH}) .{ }^{13} \mathrm{C}$ NMR (200 MHz, DMSO-d $)_{6} \delta 27.1\left(\mathrm{CH}_{2}\right), 64.6\left(\mathrm{CH}_{2}\right), 116.6$ $(\mathrm{CN}), \quad 126.2,127.6,128.9,130.8,138.1 \quad\left(\mathrm{C}_{6} \mathrm{H}_{5}\right), 157.2$ $(\mathrm{C}=\mathrm{N}), 172.5(\mathrm{C}=\mathrm{O})$. . Calculated for $\mathrm{C}_{11} \mathrm{H}_{9} \mathrm{BrN}_{4} \mathrm{O}_{3}$ : 323.99 , C: 40.64, H: 2.79, N: 17.23. Found, C: 40.40, H: 3.09, N: 17.11 .

5-(p-Cloropheny)l-2-cyanomethyl-1,3,4-oxadiazine 4a 5-(pbromo-phenyl)-2-acetonitrilo-1,3,4-oxadiazine $4 b$ and 5-(pnitrophenyl)-2-cyanomethyl-1,3,4-oxadiazine $4 c$

A suspension of either 3a (3.12 g, $0.01 \mathrm{~mol}), 3 \mathbf{b}$ (3.65 g, $0.01 \mathrm{~mol})$ or $3 \mathrm{c}(3.24 \mathrm{~g}, 0.01 \mathrm{~mol})$ in sodium ethoxide [prepared by dissolving sodium metal $(0.46 \mathrm{~g}, 0.02 \mathrm{~mol})$ in absolute ethanol $(25 \mathrm{~mL})]$ was heated in a boiling water bath for $4 \mathrm{~h}$ then left to cool. The solid product formed upon pouring into ice/water containing hydrochloric acid (till $\mathrm{pH} 7$ ) was collected by filtration.

Compound 4a: Pale yellow crystals were recovered from 1,4-dioxane in a yield of $70 \%(1.63 \mathrm{~g}) ; \mathrm{mp}: 262-265^{\circ} \mathrm{C}$. I.R $\left(\mathrm{v} / \mathrm{cm}^{-1}\right)=3060(\mathrm{CH}$ aromatic $), 2870\left(\mathrm{CH}_{2}\right), 2245(\mathrm{CN})$, $1660(\mathrm{C}=\mathrm{N}), 1638(\mathrm{C}=\mathrm{C}) .{ }^{1} \mathrm{H}$ NMR $\left(200 \mathrm{MHz}, \mathrm{DMSO}-\mathrm{d}_{6}\right)$ $\delta 4.65\left(\mathrm{~s}, 2 \mathrm{H}, \mathrm{CH}_{2}\right), 5.89\left(\mathrm{~s}, 2 \mathrm{H}\right.$, ring $\left.\mathrm{CH}_{2}\right), 7.30-7.38(\mathrm{~m}$, $\left.4 \mathrm{H}, \mathrm{C}_{6} \mathrm{H}_{4}\right) .{ }^{13} \mathrm{C}$ NMR $\left(200 \mathrm{MHz}, \mathrm{DMSO}-\mathrm{d}_{6}\right) \delta 19.8\left(\mathrm{CH}_{2}\right)$, 64.6 (ring $\left.\mathrm{CH}_{2}\right), 116.8(\mathrm{CN}), 126.0,127.3,129.9$, 130.8, $138.0\left(\mathrm{C}_{6} \mathrm{H}_{5}\right), \quad 164.2, \quad 164.8 \quad(2 \mathrm{C}=\mathrm{N})$. Calculated for $\mathrm{C}_{11} \mathrm{H}_{8} \mathrm{ClN}_{3} \mathrm{O}: 233.04, \mathrm{C}: 56.54, \mathrm{H}: 3.45, \mathrm{~N}: 17.98$. Found, C: 56.51, H: 3.60, N: 18.02 .

Compound 4b: Pale orange crystals were recovered from 1,4-dioxane in a yield of $78 \%(2.17 \mathrm{~g})$; $\mathrm{mp}: 266-269^{\circ} \mathrm{C}$. I.R $\left(\mathrm{v} / \mathrm{cm}^{-1}\right)=3066(\mathrm{CH}$ aromatic $), 2880\left(\mathrm{CH}_{2}\right), 2222(\mathrm{CN})$, $1660(\mathrm{C}=\mathrm{N}), 1635(\mathrm{C}=\mathrm{C}) .{ }^{1} \mathrm{H}$ NMR (200 MHz, DMSO-d $\left.{ }_{6}\right)$ $\delta 4.77\left(\mathrm{~s}, 2 \mathrm{H}, \mathrm{CH}_{2}\right), 5.73\left(\mathrm{~s}, 2 \mathrm{H}\right.$, ring $\left.\mathrm{CH}_{2}\right), 7.31-7.40(\mathrm{~m}$, $\left.4 \mathrm{H}, \mathrm{C}_{6} \mathrm{H}_{4}\right) .{ }^{13} \mathrm{C}$ NMR $\left(200 \mathrm{MHz}, \mathrm{DMSO}-\mathrm{d}_{6}\right) \delta 19.8\left(\mathrm{CH}_{2}\right)$, 64.6 (ring $\left.\mathrm{CH}_{2}\right), 116.6(\mathrm{CN}), 126.3,127.0,129.6,130.4$, $138.2\left(\mathrm{C}_{6} \mathrm{H}_{5}\right), \quad 164.0,164.6 \quad(2 \mathrm{C}=\mathrm{N})$. Calculated for $\mathrm{C}_{11} \mathrm{H}_{8} \mathrm{BrN}_{3} \mathrm{O}: 278.12$, C: 47.51, H: 2.90, N: 15.11. Found, C: 47.44, H: 3.30, N: 14.92 .

Compound 4c: Pale orange crystals were recovered from 1,4-dioxane in a yield of $63 \%(1.53 \mathrm{~g}) ; \mathrm{mp}: 246-249^{\circ} \mathrm{C}$. I.R $\left(\mathrm{v} / \mathrm{cm}^{-1}\right)=3066(\mathrm{CH}$ aromatic $), 2871\left(\mathrm{CH}_{2}\right), 2226(\mathrm{CN})$, $1660(\mathrm{C}=\mathrm{N}), 1639(\mathrm{C}=\mathrm{C}) .{ }^{1} \mathrm{H}$ NMR $\left(200 \mathrm{MHz}, \mathrm{DMSO}-\mathrm{d}_{6}\right)$ $\delta 4.77\left(\mathrm{~s}, 2 \mathrm{H}, \mathrm{CH}_{2}\right), 5.80\left(\mathrm{~s}, 2 \mathrm{H}\right.$, ring $\left.\mathrm{CH}_{2}\right), 7.30-7.38(\mathrm{~m}$, $\left.4 \mathrm{H}, \mathrm{C}_{6} \mathrm{H}_{4}\right)$. Calculated for $\mathrm{C}_{11} \mathrm{H}_{8} \mathrm{~N}_{4} \mathrm{O}_{3}: 244.06, \mathrm{C}: 54.10, \mathrm{H}$ : 3.30, N: 22.94. Found, C: 53.89, H: 3.36, N: 23.42. 
3-cyanomethyl-4-amino-6-phenyl-1,2,4-triazine $7 a$ and 3cyanomethyl-4-phenylamino-6-phenyl-1,2,4-triazine $7 b$

General procedure: To a solution of $3 \mathbf{a}(3.12 \mathrm{~g}, 0.01 \mathrm{~mol})$ in 1,4-dioxane $(40 \mathrm{~mL})$ either hydrazine hydrate $(0.50 \mathrm{~g}$, $0.01 \mathrm{~mol})$ or phenylhydrazine $(1.08 \mathrm{~g}, 0.01 \mathrm{~mol})$ was added. The reaction mixture was heated under reflux for $3 \mathrm{~h}$ then left to cool. The solid product formed upon pouring onto ice/water containing few drops of hydrochloric acid was collected by filtration.

Compound 7a: Yellow crystals were recovered from 1,4dioxane in a yield of $60 \%(1.48 \mathrm{~g})$; $\mathrm{mp}: 164-166^{\circ} \mathrm{C}$. I.R $\left(\mathrm{v} / \mathrm{cm}^{-1}\right)=3059(\mathrm{CH}$ aromatic $), 2890\left(\mathrm{CH}_{2}\right), 2225(\mathrm{CN})$, $1660(\mathrm{C}=\mathrm{N}), 1635(\mathrm{C}=\mathrm{C}) .{ }^{1} \mathrm{H}$ NMR $\left(200 \mathrm{MHz}, \mathrm{DMSO}-\mathrm{d}_{6}\right)$ $\delta 4.46\left(\mathrm{~s}, 2 \mathrm{H}, \mathrm{CH}_{2}\right), 4.87\left(\mathrm{~s}, 2 \mathrm{H}, \mathrm{NH}_{2}\right), 5.66(\mathrm{~s}, 2 \mathrm{H}$, triazine $\left.\mathrm{CH}_{2}\right), 7.28-7.34\left(\mathrm{~m}, 4 \mathrm{H}, \mathrm{C}_{6} \mathrm{H}_{4}\right) .{ }^{13} \mathrm{C}$ NMR $(200 \mathrm{MHz}$, DMSO-d $\left.{ }_{6}\right) \delta 19.7\left(\mathrm{CH}_{2}\right), 64.6\left(\right.$ ring $\left.\mathrm{CH}_{2}\right), 116.4(\mathrm{CN}), 126.8$, 127.3, 129.5, 129.9, $138.0\left(\mathrm{C}_{6} \mathrm{H}_{5}\right), 164.3,164.8(2 \mathrm{C}=\mathrm{N})$. Calculated for $\mathrm{C}_{11} \mathrm{H}_{10} \mathrm{ClN}_{5}: 247.06, \mathrm{C}: 53.34, \mathrm{H}: 4.07, \mathrm{~N}$ : 28.28. Found, C: $53.79, \mathrm{H}: 4.09, \mathrm{~N}: 28.39$.

Compound 7b: Yellow crystals were recovered from 1,4dioxane in a yield of $68 \%(2.19 \mathrm{~g}) ; \mathrm{mp}: 198{ }^{\circ} \mathrm{C}$. I.R $\left(\mathrm{v} / \mathrm{cm}^{-1}\right)$ $=3055(\mathrm{CH}$ aromatic $), 2883\left(\mathrm{CH}_{2}\right), 2221(\mathrm{CN}), 1662(\mathrm{C}=\mathrm{N})$, $1637(\mathrm{C}=\mathrm{C}) .{ }^{1} \mathrm{H}$ NMR $\left(200 \mathrm{MHz}, \mathrm{DMSO}-\mathrm{d}_{6}\right) \delta 4.47$ (s, $2 \mathrm{H}$, $\mathrm{CH}_{2}$ ), 4.83 (s, $2 \mathrm{H}, \mathrm{NH}_{2}$ ), 5.68 (s, $2 \mathrm{H}$, triazine $\mathrm{CH}_{2}$ ), 7.33$7.38\left(\mathrm{~m}, 4 \mathrm{H}, \mathrm{C}_{6} \mathrm{H}_{4}\right), 8.37(\mathrm{~s}, 1 \mathrm{H}, \mathrm{NH}) .{ }^{13} \mathrm{C}$ NMR $(200 \mathrm{MHz}$, DMSO-d $\left.{ }_{6}\right) \delta 19.7\left(\mathrm{CH}_{2}\right), 64.6\left(\right.$ ring $\left.\mathrm{CH}_{2}\right), 116.4(\mathrm{CN}), 126.8$, $127.3,129.5,129.9,138.0\left(\mathrm{C}_{6} \mathrm{H}_{5}\right), 164.3,164.8(2 \mathrm{C}=\mathrm{N})$. Calculated for $\mathrm{C}_{17} \mathrm{H}_{14} \mathrm{ClN}_{5}: 323.09, \mathrm{C}: 63.06, \mathrm{H}: 4.36, \mathrm{~N}$ : 21.63. Found, C: 62.88, H: 4.86, N: 21.72.

3- $\alpha$-Benzalacetonitrilo-4-amino-6-(p-chlorophenyl)-1,2,4triazine 9a, and 3- $\alpha$-benzalacetonitrilo-4-phenylamino-6(p-chlorophenyl)-1,2,4-triazine $9 b$

General Procedure: To an equimolecular amounts of dry solid of either 7a $(2.47 \mathrm{~g}, 0.01 \mathrm{~mol})$ or $7 \mathbf{b}(3.23 \mathrm{~g}, 0.01 \mathrm{~mol})$, benzaldehyde $(1.08 \mathrm{~g}, 0.01 \mathrm{~mol})$ and ammonium acetate $(1.0$ g) were added. The whole reaction mixture was heated in an oil bath at $120{ }^{\circ} \mathrm{C}$ for $15 \mathrm{~min}$ then left to cool. The solid product formed upon triturating the remaining product with ethanol was filtered off.

Compound 9a: Pale yellow crystals were recovered from acetic acid in a yield of $72 \%(2.41 \mathrm{~g}) ; \mathrm{mp}: 190-193^{\circ} \mathrm{C}$. I.R $\left(\mathrm{v} / \mathrm{cm}^{-1}\right)=3056(\mathrm{CH}$ aromatic $), 2873\left(\mathrm{CH}_{2}\right), 2220(\mathrm{CN})$, $1653(\mathrm{C}=\mathrm{N}), 1639(\mathrm{C}=\mathrm{C}) .{ }^{1} \mathrm{H}$ NMR $\left(200 \mathrm{MHz}, \mathrm{DMSO}-\mathrm{d}_{6}\right)$ $\delta 4.99\left(\mathrm{~s}, 2 \mathrm{H}, \mathrm{NH}_{2}\right), 5.64\left(\mathrm{~s}, 2 \mathrm{H}\right.$, triazine $\left.\mathrm{CH}_{2}\right), 6.61(\mathrm{~s}, 1 \mathrm{H}$, $\mathrm{CH}=\mathrm{C}), 147.2(\mathrm{CH}=\underline{\mathrm{C}}), 7.30-7.39\left(\mathrm{~m}, 9 \mathrm{H}, \mathrm{C}_{6} \mathrm{H}_{5}, \mathrm{C}_{6} \mathrm{H}_{4}\right) .{ }^{13} \mathrm{C}$ NMR (200 MHz, DMSO-d 6 ) $\delta 19.7\left(\mathrm{CH}_{2}\right), 64.6\left(\right.$ ring $\left.\mathrm{CH}_{2}\right)$, $116.8(\mathrm{CN}), 96.9(\underline{\mathrm{CH}}=\mathrm{C}), 145.7(\mathrm{CH}=\underline{\mathrm{C}}), 126.6,127.0$, $129.1,129.9,130.5,132.8,138.8\left(\mathrm{C}_{6} \mathrm{H}_{5}{ }^{\circ} \mathrm{C}_{6} \mathrm{H}_{4}\right), 164.0,164.9$ $(2 \mathrm{C}=\mathrm{N})$. Calculated for $\mathrm{C}_{18} \mathrm{H}_{14} \mathrm{ClN}_{5}: 335.09, \mathrm{C}: 64.38, \mathrm{H}$ : $4.20, \mathrm{~N}: 20.86$. Found, C: 64.54, H: 4.49, N: 21.08.

Compound 9b: Orange crystals were recovered from 1,4dioxane in a yield of $69 \%(2.83 \mathrm{~g})$; mp: $180-183^{\circ} \mathrm{C}$. I.R $\left(\mathrm{v} / \mathrm{cm}^{-1}\right)=3058(\mathrm{CH}$ aromatic $), 2876\left(\mathrm{CH}_{2}\right), 2219(\mathrm{CN})$, $1660(\mathrm{C}=\mathrm{N}), 1643(\mathrm{C}=\mathrm{C}) .{ }^{1} \mathrm{H}$ NMR (200 MHz, DMSO-d $\left.)_{6}\right)$ $\delta 5.74\left(\mathrm{~s}, 2 \mathrm{H}\right.$, triazine $\left.\mathrm{CH}_{2}\right), 6.33(\mathrm{~s}, 1 \mathrm{H}, \mathrm{CH}=\mathrm{C}), 7.27-7.38$ $\left(\mathrm{m}, 14 \mathrm{H}, 2 \mathrm{C}_{6} \mathrm{H}_{5}, \mathrm{C}_{6} \mathrm{H}_{4}\right), 8.21(\mathrm{~s}, 1 \mathrm{H}, \mathrm{NH}) .{ }^{13} \mathrm{C}$ NMR $(200$ MHz, DMSO-d 6 ) $\delta 19.9\left(\mathrm{CH}_{2}\right), 64.5\left(\right.$ ring $\left.\mathrm{CH}_{2}\right), 116.2(\mathrm{CN})$, $96.3(\underline{\mathrm{CH}}=\mathrm{C}), 145.6(\mathrm{CH}=\underline{\mathrm{C}}), 126.6,127.3,129.0,129.9$, $130.2,131.7,134.0,136.9,138.6\left(2 \mathrm{C}_{6} \mathrm{H}_{5}, \mathrm{C}_{6} \mathrm{H}_{4}\right), 164.2$,
$164.3(2 \mathrm{C}=\mathrm{N})$. Calculated for $\mathrm{C}_{24} \mathrm{H}_{18} \mathrm{ClN}_{5}: 411.13$, C: 69.98, H: 4.40, N: 17.00. Found, C: 70.24, H: 4.66, N: 16.78.

3-(Coumarin-3-yl)-4-amino-6-(p-clorophenyl)-1,2,4-triazine 11 and 3-(coumarin-3-yl)-4-phenylamino-6-(p-chlorophenyl)-1,2,4-triazine 11a

To a solution of either $7 \mathbf{a}(2.47 \mathrm{~g}, 0.01 \mathrm{~mol})$ or $7 \mathbf{b}(3.23$ $\mathrm{g}, 0.01 \mathrm{~mol})$ in 1,4 -dioxane $(40 \mathrm{~mL})$ containing piperidine $(0.5 \mathrm{~mL})$, salicylaldehyde $(1.22 \mathrm{~g}, 0.01 \mathrm{~mol})$ was added. The reaction mixture was heated under reflux for $4 \mathrm{~h}$ then evaporated under vacuum. The remaining product was triturated with ethanol and the solidified product was collected by filtration.

Compound 11a: Pale yellow crystals were recovered from acetic acid in a yield of $62 \%(2.18 \mathrm{~g})$; mp: $180-184^{\circ} \mathrm{C}$. IR $\left(v / \mathrm{cm}^{-1}\right)=3060(\mathrm{CH}$ aromatic $), 1690(\mathrm{CO}), 1673(\mathrm{C}=\mathrm{N})$, $1644(\mathrm{C}=\mathrm{C}) .{ }^{1} \mathrm{H}$ NMR $\left(200 \mathrm{MHz}, \mathrm{DMSO}_{6}\right) \delta 4.67(\mathrm{~s}, 2 \mathrm{H}$, $\left.\mathrm{NH}_{2}\right), 5.81\left(\mathrm{~s}, 2 \mathrm{H}\right.$, triazine $\left.\mathrm{CH}_{2}\right), 6.88(\mathrm{~s}, 1 \mathrm{H}$, coumarin $\mathrm{H}-4)$, 7.32-7.39 (m, 8H, 2 $\left.\mathrm{C}_{6} \mathrm{H}_{4}\right) .{ }^{13} \mathrm{C}$ NMR (200 MHz, DMSO-d $\left.\mathrm{d}_{6}\right)$ $\delta 60.5$ (ring $\mathrm{CH}_{2}$ ), 96.0 (coumarin $\mathrm{H}-4$ ), 120.3, 122.6, 124.0, $125.9,127.0,129.3,129.9,130.2,131.7,134.0,136.9,138.0$ $\left(2 \mathrm{C}_{6} \mathrm{H}_{4}\right), 159.0(\mathrm{C}=\mathrm{O}), 164.3,164.6(2 \mathrm{C}=\mathrm{N})$. Calculated for $\mathrm{C}_{18} \mathrm{H}_{13} \mathrm{ClN}_{4} \mathrm{O}_{2}$ : 354.08, C: 61.28, H: 3.71, N: 15.88. Found, C: $61.52, \mathrm{H}: 4.06, \mathrm{~N}: 16.45$.

Compound 11b: Pale brown crystals were recovered from acetic acid in a yield of $57 \%(2.43 \mathrm{~g}) ; \mathrm{mp}: 210-213^{\circ} \mathrm{C}$. I.R $\left(\mathrm{v} / \mathrm{cm}^{-1}\right)=3055(\mathrm{CH}$ aromatic $), 1688(\mathrm{CO}), 1665(\mathrm{C}=\mathrm{N})$, $1644(\mathrm{C}=\mathrm{C}) .{ }^{1} \mathrm{H}$ NMR $\left(200 \mathrm{MHz}, \mathrm{DMSO}-\mathrm{d}_{6}\right) \delta 4.42(\mathrm{~s}, 2 \mathrm{H}$, $\left.\mathrm{NH}_{2}\right), 5.77\left(\mathrm{~s}, 2 \mathrm{H}\right.$, triazine $\left.\mathrm{CH}_{2}\right), 6.90(\mathrm{~s}, 1 \mathrm{H}$, coumarin $\mathrm{H}-4)$, 7.28-7.38 (m, 8H, 2C $\left.\mathrm{C}_{6} \mathrm{H}_{4}\right) .{ }^{13} \mathrm{C}$ NMR (200 MHz, DMSO-d $\left.\mathrm{d}_{6}\right)$ $\delta 60.0$ (ring $\mathrm{CH}_{2}$ ), 96.3 (coumarin $\mathrm{H}-4$ ), 120.6, 121.9, 123.6, 125.5, 127.4, 128.7, 129.3, 130.0, 131.9, 134.8, 135.7, 138.3, 144.2, $146.9\left(\mathrm{C}_{6} \mathrm{H}_{5}, 2 \mathrm{C}_{6} \mathrm{H}_{4}\right), 158.8(\mathrm{C}=\mathrm{O}), 164.1,164.5(2$ $\mathrm{C}=\mathrm{N}$ ). Calculated for $\mathrm{C}_{24} \mathrm{H}_{17} \mathrm{ClN}_{4} \mathrm{O}_{2}: 428.10, \mathrm{C}: 67.21, \mathrm{H}$ : 4.00, N: 13.06. Found, C: 67.09, H: 3.85, N: 13.39.

\section{2- $\alpha$-5-p-chlorophenyl-1,3,4-oxadiazine 13a, 2- $\alpha$-ben- zalcyanomethyl-5-p-bromophenyl-1,3,4-oxadiazine $13 b$ and 2- $\alpha$-benzalcyanomethyl-5-p-nitrophenyl-1,3,4-oxadiazine (13c)}

General procedure: To a solution of either 3a $(3.12 \mathrm{~g}$, $0.01 \mathrm{~mol}), 3 \mathbf{b}(3.65 \mathrm{~g}, 0.01 \mathrm{~mol}), 3 \mathbf{c}(3.24 \mathrm{~g}, 0.01 \mathrm{~mol}), 4 \mathbf{a}$ $(2.33 \mathrm{~g}, 0.01 \mathrm{~mol}), \mathbf{4 b}(2.79 \mathrm{~g}, 0.01 \mathrm{~mol})$ or $4 \mathbf{c}(2.44 \mathrm{~g}, 0.01$ $\mathrm{mol})$ in 1,4-dioxane $(40 \mathrm{~mL})$ containing piperidine $(1.0 \mathrm{~mL})$ benzaldehyde $(1.08 \mathrm{~g}, 0.01 \mathrm{~mol})$ was added. The reaction mixture was heated under reflux for $6 \mathrm{~h}$ then poured onto ice/water containing few drops of hydrochloric acid. The solid product, formed in each case, was collected by filtration to afford the respective products.

Compound 13a: Yellow crystals were recovered from methanol in a yield of $63 \%(2.02 \mathrm{~g})$, from 3a and $70 \%$ (2.25) from 4a; mp: $180-182^{\circ} \mathrm{C}$. I.R $\left(\mathrm{v} / \mathrm{cm}^{-1}\right)=3055(\mathrm{CH}$ aromatic), $2220(\mathrm{CN}), 1656(\mathrm{C}=\mathrm{N}), 1640(\mathrm{C}=\mathrm{C}) .{ }^{1} \mathrm{H}$ NMR $\left(200 \mathrm{MHz}, \mathrm{DMSO}-\mathrm{d}_{6}\right) \delta 5.60\left(\mathrm{~s}, 2 \mathrm{H}\right.$, oxadiazine $\left.\mathrm{CH}_{2}\right), 6.30$ (s, $1 \mathrm{H}, \mathrm{CH}=\mathrm{C}$ ), 7.30-7.39(m, 9H, $\left.\mathrm{C}_{6} \mathrm{H}_{5}, \mathrm{C}_{6} \mathrm{H}_{4}\right) .{ }^{13} \mathrm{C}$ NMR (200 MHz, DMSO-d $\left.{ }_{6}\right) \delta 60.4$ (ring $\left.\mathrm{CH}_{2}\right), 95.0(\mathrm{CH}=\mathrm{C})$, $118.0(\mathrm{CH}=\underline{\mathrm{C}}), 120.3,121.5,123.2,125.0,127.4,129.3$, $130.0,134.8,135.0,144.0\left(\mathrm{C}_{6} \mathrm{H}_{5}, \mathrm{C}_{6} \mathrm{H}_{4}\right), 164.0,164.2(2$ $\mathrm{C}=\mathrm{N}$ ). Calculated for $\mathrm{C}_{18} \mathrm{H}_{12} \mathrm{ClN}_{3} \mathrm{O}: 322.06, \mathrm{C}: 67.19, \mathrm{H}$ : 3.76, N: 13.06. Found, C: 67.33, H: 4.06, N: 12.74 . 
Compound 13b: Orange-red crystals were recovered from methanol/dioxane mixture in a yield of $66 \%(2.41 \mathrm{~g})$; mp: $210-212^{\circ} \mathrm{C}$. I.R $\left(\mathrm{v} / \mathrm{cm}^{-1}\right)=3060(\mathrm{CH}$ aromatic $), 2226$ $(\mathrm{CN}), 1665(\mathrm{C}=\mathrm{N}), 1621 \quad(\mathrm{C}=\mathrm{C}) .{ }^{1} \mathrm{H} \mathrm{NMR}(200 \mathrm{MHz}$, DMSO-d $\left.{ }_{6}\right) \delta 5.77\left(\mathrm{~s}, 2 \mathrm{H}\right.$, oxadiazine $\left.\mathrm{CH}_{2}\right), 6.20(\mathrm{~s}, 1 \mathrm{H}$, $\mathrm{CH}=\mathrm{C}), 7.29-7.39\left(\mathrm{~m}, 9 \mathrm{H}, \mathrm{C}_{6} \mathrm{H}_{5}, \mathrm{C}_{6} \mathrm{H}_{4}\right) .{ }^{13} \mathrm{C} \mathrm{NMR}(200$ $\left.\mathrm{MHz}, \mathrm{DMSO}-\mathrm{d}_{6}\right) \delta 60.6\left(\operatorname{ring} \mathrm{CH}_{2}\right), 95.2(\underline{\mathrm{CH}}=\mathrm{C}), 118.3$ $(\mathrm{CH}=\underline{\mathrm{C}}), 120.0,120.2,121.6,123.2,127.4,128.0,130.2$, 132.6, 134.2, $140.4\left(\mathrm{C}_{6} \mathrm{H}_{5}, \mathrm{C}_{6} \mathrm{H}_{4}\right), 164.1,164.8(2 \mathrm{C}=\mathrm{N})$. Calculated for $\mathrm{C}_{18} \mathrm{H}_{12} \mathrm{BrN}_{3} \mathrm{O}: 365.02, \mathrm{C}: 59.03, \mathrm{H}: 3.30, \mathrm{~N}$ : 11.47. Found, C: $58.92, \mathrm{H}: 3.55, \mathrm{~N}: 11.51$.

Compound 13c: Orange crystals were recovered from 1,4-dioxane in a yield of $70 \%(2.32 \mathrm{~g}) ; \mathrm{mp}: 233-236^{\circ} \mathrm{C}$. I.R $\left(\mathrm{v} / \mathrm{cm}^{-1}\right)=3063(\mathrm{CH}$ aromatic $), 2223(\mathrm{CN}), 1656(\mathrm{C}=\mathrm{N})$, $1638(\mathrm{C}=\mathrm{C}) .{ }^{1} \mathrm{H}$ NMR $\left(200 \mathrm{MHz}, \mathrm{DMSO}-\mathrm{d}_{6}\right) \delta 5.80(\mathrm{~s}, 2 \mathrm{H}$, ring $\left.\mathrm{CH}_{2}\right), 6.21(\mathrm{~s}, 1 \mathrm{H}, \mathrm{CH}=\mathrm{C}), 7.32-7.46\left(\mathrm{~m}, 9 \mathrm{H}, \mathrm{C}_{6} \mathrm{H}_{4}\right) .{ }^{13} \mathrm{C}$ NMR (200 MHz, DMSO-d $\left.{ }_{6}\right) \quad \delta 60.3 \quad$ (ring $\mathrm{CH}_{2}$ ), 95.5 $(\underline{\mathrm{CH}}=\mathrm{C}), 118.1(\mathrm{CH}=\underline{\mathrm{C}}), 120.0,120.5,121.6,123.2,125.9$, 126.8, 130.8, 132.9, 133.9, $141.0\left(\mathrm{C}_{6} \mathrm{H}_{5}, \mathrm{C}_{6} \mathrm{H}_{4}\right), 164.3,164.5$ $(2 \mathrm{C}=\mathrm{N})$. MS: $\mathrm{m} / \mathrm{e}=332\left(\mathrm{M}^{+}\right)$. Calculated for $\mathrm{C}_{18} \mathrm{H}_{12} \mathrm{~N}_{4} \mathrm{O}_{3}$ : 332.09, C: 65.06, H: 3.64, N: 16.86. Found, C: 64.88, H: 4.42, $\mathrm{N}: 16.55$.

5-Amino-1-cyanoacetyl-3-(p-chlorophenyl)-pyrazole 15a, 5-Amino-1-cyanoacetyl-3-(p-bromophenyl)-pyrazole $15 b$ and 5-Amino-1-cyanoacetyl-3-(p-nitrophenyl)-pyrazole $15 \mathrm{c}$

General procedure: To a solution of either 3a (3.12 g, $0.01 \mathrm{~mol}), 3 \mathbf{b}(3.65 \mathrm{~g}, 0.01 \mathrm{~mol})$ or $3 \mathbf{c}(3.24 \mathrm{~g}, 0.01 \mathrm{~mol})$ in ethanol $(40 \mathrm{~mL})$, a solution of potassium cyanide $(2.8 \mathrm{~g}, 0.05$ mol) was added. The reaction mixture, in each case, was heated in a warm water bath at $60{ }^{\circ} \mathrm{C}$ for $0.5 \mathrm{~h}$ then left with stirring at room temperature overnight. The solid product formed upon pouring onto ice/water containing hydrochloric acid (till pH 6) was collected by filtration.

Compound 15a: Orange crystals were recovered from 1,4-dioxane in a yield of $74 \%(1.92 \mathrm{~g})$; mp: $175-178^{\circ} \mathrm{C}$. I.R $\left(\mathrm{v} / \mathrm{cm}^{-1}\right)=3460-3377\left(\mathrm{NH}_{2}\right), 3050(\mathrm{CH}$ aromatic $), 2250$ $(\mathrm{CN}), 1688(\mathrm{C}=\mathrm{O}), 1650(\mathrm{C}=\mathrm{N}), 1636(\mathrm{C}=\mathrm{C}) .{ }^{1} \mathrm{H}$ NMR $(200$ $\left.\mathrm{MHz}, \mathrm{DMSO}-\mathrm{d}_{6}\right) \delta 4.64\left(\mathrm{~s}, 2 \mathrm{H}, \mathrm{CH}_{2}\right), 4.90\left(\mathrm{~s}, 2 \mathrm{H}, \mathrm{NH}_{2}\right)$, 6.52 (s, 1H, pyrazole $\mathrm{H}-4), 7.28-7.34\left(\mathrm{~m}, 4 \mathrm{H}, \mathrm{C}_{6} \mathrm{H}_{4}\right) .{ }^{13} \mathrm{C}$ NMR (200 MHz, DMSO-d $\left.{ }_{6}\right) \quad \delta 60.6$ (ring $\left.\mathrm{CH}_{2}\right), 95.2$ $(\underline{\mathrm{CH}}=\mathrm{C}), 118.0(\mathrm{CH}=\underline{\mathrm{C}}), 120.3,120.9,121.4,123.0,124.9$, 126.9, 138.8, 130.9, 133.9, $140.2\left(\mathrm{C}_{6} \mathrm{H}_{5}, \mathrm{C}_{6} \mathrm{H}_{4}\right), 164.2,164.8$ $(2 \mathrm{C}=\mathrm{N})$. MS: $\mathrm{m} / \mathrm{e}=260\left(\mathrm{M}^{+}\right)$. Calculated for $\mathrm{C}_{12} \mathrm{H}_{9} \mathrm{ClN}_{4} \mathrm{O}$ : 260.05, C: 55.29, H: 3.48, N: 21.49. Found, C: 55.43, H: $3.68, \mathrm{~N}: 21.26$.

Compound 15b: Brown crystals were recovered from 1,4-dioxane in a yield of $70 \%(2.13 \mathrm{~g})$; mp: of $177-180^{\circ} \mathrm{C}$. I.R $\left(\mathrm{v} / \mathrm{cm}^{-1}\right)=3455-3349\left(\mathrm{NH}_{2}\right), 3055(\mathrm{CH}$ aromatic $), 2243$ $(\mathrm{CN}), 1690(\mathrm{C}=\mathrm{O}), 1655(\mathrm{C}=\mathrm{N}), 1638(\mathrm{C}=\mathrm{C}) .{ }^{1} \mathrm{H}$ NMR $(200$ $\left.\mathrm{MHz}, \mathrm{DMSO}-\mathrm{d}_{6}\right) \delta 4.65\left(\mathrm{~s}, 2 \mathrm{H}, \mathrm{CH}_{2}\right), 4.75\left(\mathrm{~s}, 2 \mathrm{H}, \mathrm{NH}_{2}\right)$, $6.49(\mathrm{~s}, 1 \mathrm{H}$, pyrazole $\mathrm{H}-4), 7.29-7.38\left(\mathrm{~m}, 4 \mathrm{H}, \mathrm{C}_{6} \mathrm{H}_{4}\right) .{ }^{13} \mathrm{C}$ NMR (200 MHz, DMSO-d $\left.{ }_{6}\right) \quad \delta 60.3 \quad\left(\right.$ ring $\left.\mathrm{CH}_{2}\right), 95.2$ $(\underline{\mathrm{CH}}=\mathrm{C}), 118.4(\mathrm{CH}=\underline{\mathrm{C}}), 120.6,120.3,121.0,123.3,124.7$, $126.9,138.8,130.9,133.9,140.9\left(\mathrm{C}_{6} \mathrm{H}_{5}, \mathrm{C}_{6} \mathrm{H}_{4}\right), 164.0,164.8$ (2 $\mathrm{C}=\mathrm{N})$. Calculated for $\mathrm{C}_{12} \mathrm{H}_{9} \mathrm{BrN}_{4} \mathrm{O}: 305.13, \mathrm{C}: 47.24, \mathrm{H}$ : 2.97, N: 18.36. Found, C: 47.08, H: 3.22, N: 18.73.

Compound 15c: Dark orange crystals were recovered from 1,4-dioxane in a yield of $64 \%(1.73 \mathrm{~g})$; mp: 195-199 ${ }^{\circ}$ C. I.R $\left(\mathrm{v} / \mathrm{cm}^{-1}\right)=3459-3354\left(\mathrm{NH}_{2}\right), 3052(\mathrm{CH}$ aromatic $)$, $2239(\mathrm{CN}), 1688(\mathrm{C}=\mathrm{O}), 1657(\mathrm{C}=\mathrm{N}), 1636(\mathrm{C}=\mathrm{C}) .{ }^{1} \mathrm{H}$ NMR
(200 MHz, DMSO-d 6 ) $\delta 4.67\left(\mathrm{~s}, 2 \mathrm{H}, \mathrm{CH}_{2}\right), 4.79\left(\mathrm{~s}, 2 \mathrm{H}, \mathrm{NH}_{2}\right)$, $6.52(\mathrm{~s}, 1 \mathrm{H}$, pyrazole $\mathrm{H}-4), 7.29-7.36\left(\mathrm{~m}, 4 \mathrm{H}, \mathrm{C}_{6} \mathrm{H}_{4}\right) .{ }^{13} \mathrm{C}$ NMR (200 MHz, DMSO-d 6 ) $\delta 60.3 \quad\left(\right.$ ring $\left.\mathrm{CH}_{2}\right), 95.2$ $(\underline{\mathrm{CH}}=\mathrm{C}), 118.0(\mathrm{CH}=\mathrm{C}), 120.3,120.9,121.4,123.0,124.9$, 126.9, 138.8, 130.9, 133.9, $138.2\left(\mathrm{C}_{6} \mathrm{H}_{5}, \mathrm{C}_{6} \mathrm{H}_{4}\right), 164.5,164.7$ $(2 \mathrm{C}=\mathrm{N})$. MS: $\mathrm{m} / \mathrm{e}=271\left(\mathrm{M}^{+}\right)$. Calculated for $\mathrm{C}_{12} \mathrm{H}_{9} \mathrm{~N}_{5} \mathrm{O}_{3}$ : 271.07, C: 53.14, H: 3.34, N: 25.82. Found, C: 52.88, H: $3.20, \mathrm{~N}: 25.43$.

\section{ACKNOWLEDGEMENT}

R. M. Mohareb thanks the Alexander von Humboldt Foundation for the financial support during summer, 2009 during his fellowship in Germany (Erlangen) for doing research and completing this work.

\section{REFERENCES}

[1] Rallas, S.; Gulerman, N.; Erdeniz, H. Synthesis and antimicrobial activity of some new hydrazones of 4-fluorobenzoic acid hydrazide and 3-acetyl-2,5-disubstituted-1,3,4-oxadiazolines. II FARMACO, 2002, 57(2), 171-174.

[2] Gursoy, A.; Terzioglu, N.; Otuk, G. Synthesis of some new hydrazide-hydrazones, thiosemicarbazides and thiazolidinones as possible antimicrobials. Eur. J. Med. Chem., 1997, 32, 753-757.

[3] Vicini, P.; Zani, F.; Cozzini, P.; Doytchinova, I. Hydrazones of 1,2benzisothiazole hydrazides: synthesis, antimicrobial activity and QSAR investigations. Eur. J. Med. Chem., 2002, 37, 553-564.

[4] Mamolo, M.G.; Falagiani, V.; Zampieri, D.; Vio, L.; Banfo, E. Synthesis and antimycobacterial activity of [5-(pyridin-2-yl)-1,3,4thiadiazol-2-ylthio]acetic acid arylidene-hydrazide derivatives. II FARMACO, 2001, 56, 587-592.

[5] Rahman, V.M.; Mukhtar, S.; Ansari, W.H.; Lemiere, G. Syhthesis, stereochemistry and biological activity of some novel long alkyl chain substituted thiazolidin-4-ones and thiazan-4-one from 10undecenoic acid hydrazide. Eur. J. Med. Chem., 2005, 40, 173-184.

[6] Dimmock, J.R.; Vashishtha, S. C.; Stables, J.P. Anticonvulsant properties of various acetylhydrazones, oxamoylhydrazones and semicarbazones derived from aromatic and unsaturated carbonyl compounds. Eur. J. Med. Chem., 2000, 35, 241-248.

[7] Yapia, R.; La Mara, M.P., Massieu, G.H. Modifications of brain glutamate decarboxylase activity by pyridoxal phosphate- $\gamma$ glutamyl hydrazone. Biochem. Pharmacol, 1967, 16(7), 1211-1218.

[8] Sava, G.; Perissin, L.; Lassiani, L.; Zabucchi, G. Antiinflammatory action of hydrosoluble dimethyl-triazenes on the carrageenininduced edema in guinea pigs. Chem. Biol. Interact., 1985, 53, 3743.

[9] Xia, Y.1; Chuan-Dong, F.; Zhao, B.X.; Zhao, J.; Shin, D.S.; Miaom J.Y. Synthesis and structure-activity relationships of novel 1arylmethyl-3-aryl-1H-pyrazole-5-carbohydrazide hydrazone derivatives as potential agents A549 lung cancer cells. Eur. J. Med. Chem., 2008, 43, 2347-2353.

[10] Melnyk, P.; Leroux, V.; Serghergert, C.; Grellier, P. Design, synthesis and in vitro antimalarial activity of an acylhydrazone library. Bioorg. Med. Chem. Lett., 2006, 16, 31-35.

[11] Ajani, O.O.; Obafemi, C.A.; Nwinyi, O.C.; Akinpelu, D.A. Microwave assisted synthesis and antimicrobial activity of 2quinoxalinone-3-hydrazone derivastives. Bioorg. Med. Chem., 2010, 18, 214-221.

[12] Zheng, L.W.; Wu, L.L.; Zhao, B.X.; Dong, W.L.; Miao, Y.J. Synthesis of novel substituted pyrazole-5-carbohydrazide hydrazone derivatives and discovery of a potent apoptosis inducer in A549 lung cancer cells. Bioorg. Med. Chem., 2009, 17, 1957-1962

[13] Bhagavan, N.V. In: Medical Biochemistry; Elsevier Science B. V: Amsterdam, 2002; Vol. 17, pp. 331-363.

[14] Saulnier, M.G.; Velaprthi, U.; Zimmermann, K. In: Progress In Heterocyclic Synthesis; Gorden Gribble, Ed.; Elsevier Science B.V.: Amsterdam, 2005; Vol. 16, pp. 228-271.

[15] Short, E.I. Studies on the inactivation of isonicotinyl acid hydrazide in normal subjects and tuberculous patients. Tubercle, 1962, 43, 33-42.

[16] Holdiness, M.R. A review of blood dyecrasias induced by the antituberculosis drugs. Tubercle, 1987, 68, 301-309.

[17] Faroumadi, A.; Kiano, Z.; Soltani, F. Antituberculosis agents VIII: Synthesis and in vitro antimycobacterial activity of alkyl $\alpha-[5-(5-$ 
nitro-2-thienyl)-1,3,4-thiadiazole-2-ylthio]acetates. II FARMACO, 2003, 58, 1073-1076.

[18] Bokharev, V.V.; Ghidaspov, A.A.; Peresedova, E.V. Reaction of potassium salts of 2-amino-4-methoxy-6-dinitro-methyl-1,3,5triazines with $\mathrm{N}_{2} \mathrm{O}_{4}$. Chem. Heterocycl. Comp., 2006, 42, 10961106.

[19] Riddell, F.G.; E.; Turner, E.S. Conformational equilibria and nitrogen inversion in tetrahydro-1,2,5-oxadiazines. Tetrahedron, 1979, $35,1311-1314$.
[20]

Brzozowski, Z; Czewski, F.S. Synthesis and antitumor activity of novel 2-amino-4-(3,5,5-trimethyl-2-pyrazolino)-1,3,5-triazine derivatives. Eur. J. Med. Chem., 2002, 37, 709-720.

[21] Sherif, A., Rostom, F. Polysubstituted pyrazoles, part 6. Synthesis of some 1-(4-chlorophenyl)-4-hydroxy-1H-pyrazol-3-carbonyl derivatives linked to nitrogenous heterocyclic ring systems as potential antitumor agents. Bioorg. Med. Chem., 2010, 18, 2767-2776.

Received: February 19, 2010

(C) Mohareb et al.; Licensee Bentham Open.

This is an open access article licensed under the terms of the Creative Commons Attribution Non-Commercial License (http://creativecommons.org/licenses/by-nc/3.0/) which permits unrestricted, non-commercial use, distribution and reproduction in any medium, provided the work is properly cited. 